\title{
MANNA-WUI: Um nó sensor versátil para a Internet das Coisas
}

\author{
Wuigor I. S. Bine ${ }^{1}$, Linnyer Beatrys Ruiz ${ }^{1}$ \\ ${ }^{1}$ Programa de Pós-Graduação em Ciência da Computação (PCC) \\ Universidade Estadual de Maringá (UEM) \\ Maringá - Paraná - Brasil \\ wugor@hotmail.com, lbruiz@uem.br
}

\begin{abstract}
The goal of this paper is to introduce and to evaluate the MANNAWUI device performance in urban and non-urban environments in terms of data transmission maximum distance and energy consumption. This device is a generic sensor node that provides greater processing capacity and uses LoRa technology for long-distance transmissions. It is based on the challenges of the Internet of Things area, which involve the installation of smart objects in nonurban areas, and the low processing capacity in the existing ones. The results of the tests performed were satisfactory for data transmission over long distances in non-urban areas reaching $18 \mathrm{~km}$ and its energy consumption in the sleep mode of $13,2 \mu \mathrm{W}$.
\end{abstract}

Resumo. O objetivo deste trabalho é apresentar e avaliar a performance do dispositivo MANNA-WUI nos ambientes urbanos e não urbanos em termos da distância máxima alcançada para transmissão de dados e consumo energético. Este dispositivo é um nó sensor genérico que fornece maior capacidade de processamento e utiliza tecnologia LoRa para transmissões de longa distância. Ele baseia-se nos desafios da área de Internet das Coisas, que envolvem a instalação de objetos inteligentes em áreas não urbanas, e a pouca capacidade de processamento nos existentes. Os resultados dos testes realizados foram satisfatórios para transmissão de dados a longa distância em áreas não-urbanas chegando a $18 \mathrm{Km}$ e seu consumo energético no modo sleep de 13,2 $\mu \mathrm{W}$.

\section{Introdução}

A Internet das Coisas (IoT) é um paradigma que tem um espaço bem consolidado no cenário de redes sem fio. Seu desenvolvimento foi possibilitado por conta dos avanços obtidos na última década com sensores inteligentes, processadores e transceptores sem fio de baixo consumo, sistemas embarcados e protocolos de comunicação que, juntos, a promovem.

A estrutura da IoT pode ser aplicada em cidades e casas inteligentes, transporte, veículos, escolas, serviços de saúde e agricultura [Kjellby et al. 2018]. Essas aplicações vêm revolucionando a forma de como lidamos com tarefas do nosso cotidiano e auxiliando na tomada de decisões em nosso trabalho. [Gartner 2018] prevê que em 2021 teremos mais de 25 bilhões de dispositivos de IoT. Empresas como Ericsson e Cisco estimam cerca de 50 bilhões de dispositivos conectados à internet [Lee and Lee 2015, Derhamy et al. 2015]. 
Os dispositivos que compõem a Internet das Coisas comumente conhecidos como objetos inteligentes são equipados com sensores, atuadores, unidades de processamento, memória, comunicação e bateria. Esta composição permite com que o nó seja responsável pela coleta de dados e armazenamento. Este universo vêm permitindo a combinação dos dispositivos IoT com smartphones, possibilitando realizar ações com os objetos inteligentes, tornando-os cada vez mais populares [Serikul et al. 2018].

Pesquisadores estão dedicando esforços no desenvolvimento de aplicações para áreas não urbanas, destacando o setor de agricultura. Por exemplo, no trabalho de [Serikul et al. 2018] é desenvolvido um sistema para monitoramento inteligente de umidade para fazendas. Nesse caso, é utilizada uma placa de prototipagem que é equipada com um ESP8266-12E para construção do nó. Já em [Baldovino et al. 2018], o autor realiza a implementação de uma rede de sensores sem fio de baixa potência para aplicações agrícolas. Na construção do nó sensor, o autor utilizou duas placas comercialmente encontradas sendo, Arduino Mega como placa de controle junto com o ESP8266. No trabalho de [Adam et al. 2019], que propõe um sistema de agricultura preditiva, também são utilizados para construção dos nós sensores o ESP8266.

Boa parte dos pesquisadores optam por utilizar placas de desenvolvimentos disponíveis no mercado para construção de seus próprios nós sensores devido ao custo acessível e a facilidade que os mesmos proporciona. Entretanto, a escolha da unidade de processamento, que comumente é um microcontrolador, deve levar em consideração a aplicação a ser construída em um equilíbrio com o consumo de energia, para não prejudicar o desempenho de vida útil da mesma. Além disso, nem sempre essas placas atendem o requisito de serem de baixo consumo e, ademais, não realizam o monitoramento da bateria.

Construir um nó para aplicações IoT que se encaixe em especificações rigorosas de autonomia energética e capacidade de processamento exige etapas de análises em duas unidades principais, a saber, de comunicação e processamento. Existem diferentes tecnologias de comunicação por RF já consolidadas comercialmente e academicamente para serem utilizadas. Há uma série de pesquisas que se dedicam a explorar essas tecnologias de transmissão, entre elas: Zigbee [Fitriawan et al. 2017, Lin and Wang 2017, Edward Jero and Balaji Ganesh 2011], NB-IoT [Wang et al. 2019, Duangsuwan et al. 2018, Jörke et al. 2018] e LoRa [Mayer et al. 2019, Addabbo et al. 2019, Bouguera et al. 2018].

Quando se trata de áreas não urbanas é importante que o consumo de energia seja baixo e que a tecnologia de comunicação por radiofrequência (RF) seja de longo alcance (Low Power Wide Area Network - LPWAN). Essas configurações do dispositivo estão diretamente relacionadas à autonomia do mesmo, já que o ecossistema da IoT prevê centenas de milhares de nós comunicando com o mínimo de interferência humana.

Este trabalho tem como objetivo apresentar e avaliar a performance do MANNAWUI ${ }^{1}$ : um nó sensor para o paradigma de Internet das Coisas. O nó desenvolvido tem como prioridade a autonomia energética e o alcance de comunicação. As decisões relacionadas à unidade de processamento levam em consideração o desenvolvimento de

\footnotetext{
${ }^{1}$ MANNA: Laboratório de Engenharia de Computação Invisível da Universidade Estadual de Maringá http://manna.team/
} 
um dispositivo mais robusto e com capacidades acima das comumente encontradas em dispositivos de baixo consumo de energia.

O artigo está organizado da maneira que segue: a Seção 2 apresenta os trabalhos relacionados. Em seguida, a Seção 3 apresenta o projeto e implementação do nó sensor, assim como seus subsistemas. A Seção 4 apresenta os custos para construção do nó sensor e a implementação do algoritmo para realização de testes. A Seção 5 apresenta os resultados obtidos na avaliação da performance do dispositivo em termos de distâncias para ambientes urbanos e não urbanos e o consumo energético do nó sensor. Finalizamos com a Seção 6 concluindo os resultados obtidos do trabalho.

\section{Trabalhos Relacionados}

Trabalhos recentes, sumarizados na Tabela 1, apresentam a importância de se desenvolver um protótipo levando aspectos de baixo consumo. Como apresentado no trabalho de [Mayer et al. 2019], foi desenvolvido um nó sensor levando em consideração aspectos de baixo consumo, onde se utilizou um microcontrolador Cortex-M4 de baixa potência e realizou análise do consumo de corrente entre dois transceptores LoRa sendo o SX1276 e SX1261. Com o foco principal em mostrar a diferença entre os transceptores, o autor apresenta a diferença de potência em cada modo de operação do transceptor chamando a atenção para o modo de transmissão onde se obteve uma redução de $74 \%$ em relação ao SX1276.

Tabela 1. Comparação dos trabalhos relacionados com o MANNA-WUI

\begin{tabular}{|l|l|l|l|l|}
\hline \multicolumn{1}{|c|}{ Trabalho } & Microcontrolador & $\begin{array}{c}\text { Monitoramento } \\
\text { da bateria }\end{array}$ & $\begin{array}{c}\text { Transceptores } \\
\text { onboard }\end{array}$ & \multicolumn{1}{c|}{$\begin{array}{c}\text { Desligamento } \\
\text { de subsistemas } \\
\text { (sensores/transceptores) }\end{array}$} \\
\hline [Mayer et al. 2019] & STM32L475 & Sim & LoRa & Não \\
\hline [Magno et al. 2017] & MSP430 & Não & LoRa & Não \\
\hline [Tokmakov et al. 2019] & ATMEGA328 & Não & LoRa & Não \\
\hline MANNA-WUI & STM32F407 & Sim & LoRa, Wi-Fi, BLE & Sim \\
\hline
\end{tabular}

O autor [Magno et al. 2017], propõem um nó final para IoT com eficiência energética utilizando um microcontrolador MSP430 de arquitetura RISC de 16-Bits e o transceptor LoRa SX1276. O protótipo apresentou um resultado na transmissão com potência de saída a $14 \mathrm{dBm}$ um consumo de $381,1 \mathrm{~mW}$ e para $10 \mathrm{dBm}$ o resultado foi de $281,2 \mathrm{~mW}$.

[Tokmakov et al. 2019] apresenta o design de um nó de borda de ultra baixa potência utilizando tecnologia de transmissão LoRaWAN. O nó sensor é equipado com apenas um único sensor (BMP280) que faz medições de temperatura e pressão. O dispositivo é alimentado por duas baterias AA que permitem que o sistema funcione de forma autônoma. O diferencial do projeto é a utilização de um circuito integrado (TPL5110) que realiza a função de acordar o microcontrolador (ATMEGA328) para coletar dados do sensor e realizar o envio de uma mensagem. O autor apresenta que ao utilizar o TPL5110 ao invés do WDT (Watchdog Timer) do microcontrolador para contagem do tempo o disparo do sinal para sair do modo sleep, consegue-se uma redução no consumo de corrente de 3,91 $\mu \mathrm{A}$, ficando em $290 \mathrm{nA}$ e na leitura dos sensores e modo ativo do dispositivo uma redução de $0,7 \mathrm{~mA}$, chegando a 3,6 mA. 
O MANNA-WUI propõe um nó sensor versátil, onde seus sensores e transmissores não interfira em aplicações específicas onde não serão utilizados. Os sensores e transmissores (Wi-Fi, Bluetooth e LoRa) estão presentes na placa sem a necessidade de utilização de módulos externos e permitem seu desligamento da fonte de energia, caso não sejam utilizados. Junto com o emprego de um microcontrolador mais robusto permite realizar processamento na borda e envio de dados já processados ou pré processados.

\section{Projeto e implementação do sistema}

Esta Seção descreve o projeto e implementação do MANNA-WUI, que consiste em um nó sensor para IoT, tendo como prioridade a autonomia energética e alcance de comunicação. O dispositivo foi desenvolvido de modo que todos os nós sensores são idênticos e possuem os mesmo periféricos, proporcionando que qualquer nó sensor seja utilizado como gateway ou permitindo mais de um ponto de saída de dados para nuvem, dependendo do tipo de topologia de rede empregada.

Outra ponto importante, é que caso ocorra falhas na rede, outro nó sensor próximo a uma rede Wi-Fi pode assumir a posição do gateway. O nó sensor foi projetado utilizando o microcontrolador ARM Cortex-M4 da empresa STMicroelectronics, especificamente o STM32F407VG. Este microcontrolador possui uma unidade FPU, DSP, 1 Mbyte Flash e pode operar com até $168 \mathrm{MHz}$.O esquemático simplificado do nó sensor é apresentado na Figura 1.

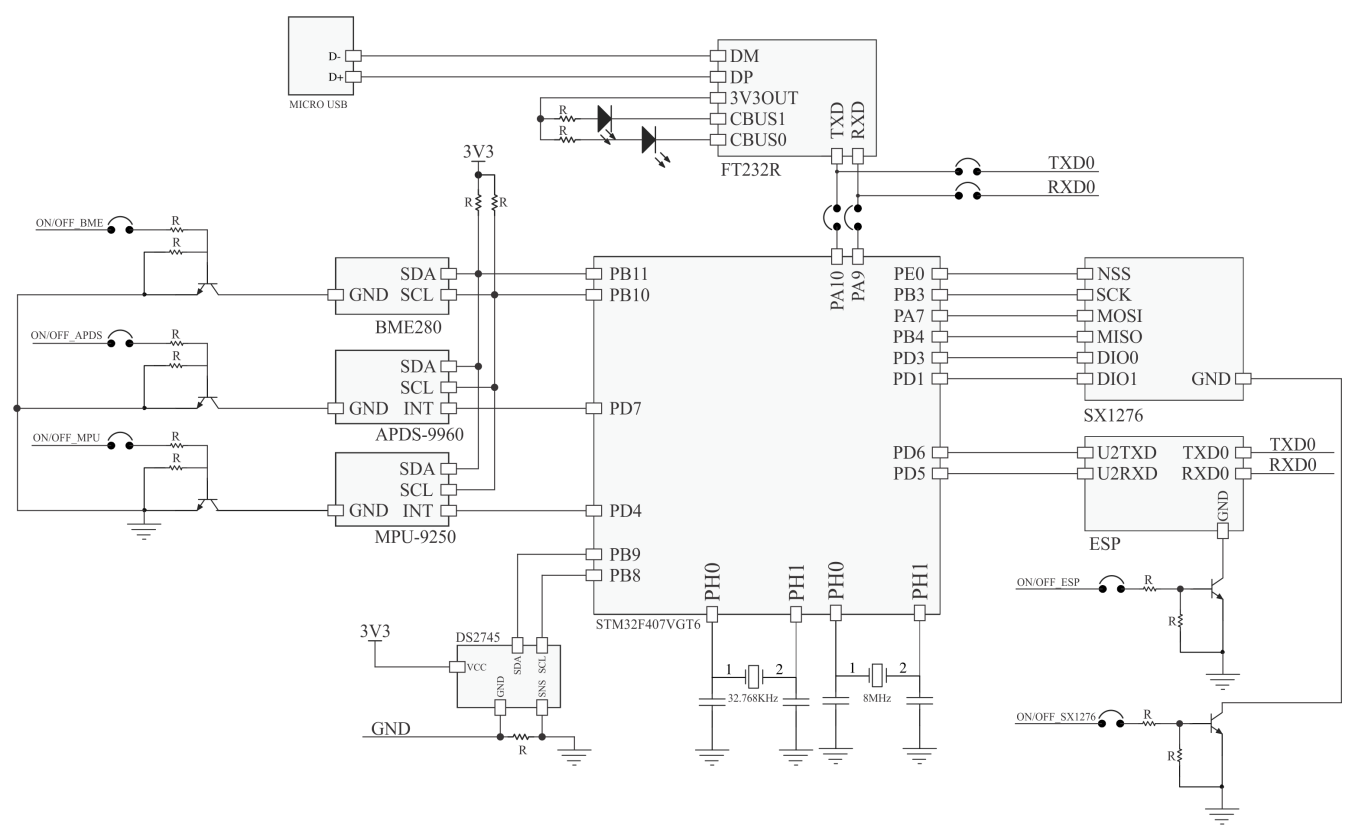

Figura 1. Esquemático resumido do circuito do nó sensor MANNA-WUI.

A escolha do microcontrolador Cortex-M4 foi feita para abranger os dois principais cenários do IoT, urbanos e não urbanos. Em ambos os locais, principalmente em ambientes urbanos o processamento de imagem está cada vez mais presente. O microcontrolador possui interface DCMI para comunicação com câmeras de até 12-Bits. Propor um nó sensor um pouco fora dos padrões tradicionais tendo como principal diferencial a utilização de um microcontrolador mais robusto, mas levando em consideração todos 
os aspectos para se ter o menor consumo energético, permite realizar processamento na borda e enviando dados já processados ou pré processados.

Em sua interface de transmissão de dados de longo alcance é utilizado o transceptor SX1276 [SEMTECH 2019] com tecnologia LoRa. Para realizar a comunicação Wi-Fi é utilizado o transceptor ESP32-DOWDQ6 [ESPRESSIF 2019] que também pode operar como um transceptor bluetooth. Os sensores que foram utilizados são o BME280 [BOSCH 2018] (temperatura, pressão, umidade), MPU9250 [InvenSense 2016] (giroscópio, acelerômetro e magnetômetro) e APDS9960 (monitoramento de luz e movimento). Todos os sensores escolhidos para este dispositivo são de baixo consumo energético.

O monitoramento da bateria é de extrema importância para que o nó sensor opere de forma inteligente na tomada de decisões de forma automatizadas de modo a prolongar a vida útil da bateria. Assim, foi projetado o circuito de monitoramento utilizando o DS2745 [Dallas Semiconductor 2009] que possui um consumo energético no modo ativo de $70 \mu \mathrm{A}$ e em seu modo sleep $1 \mu \mathrm{A}$.

A Figura 2 apresenta o protótipo do nó sensor proposto que, além dos periféricos mencionados, também dispõe de uma interface USB-Serial, permitindo realizar o upload de firmware para o MCU e facilitando a configuração do nó sensor para o usuário final via comandos AT.

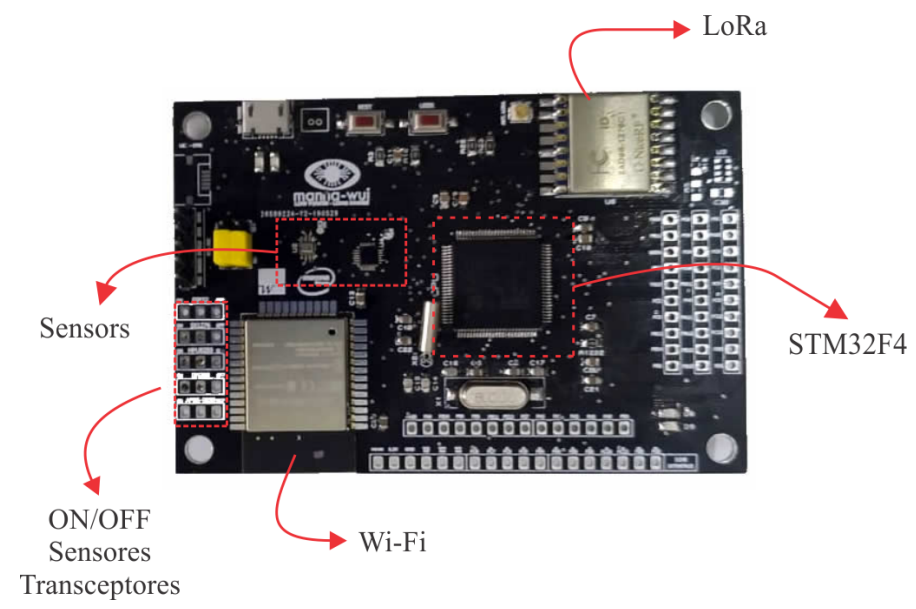

Figura 2. Protótipo MANNA-WUI para transmissões de longas distâncias.

\subsection{Monitoramento da bateria}

A etapa de monitoramento da bateria é o fator mais importante quando se trata de maximizar sua durabilidade. Com os dados coletados da bateria podemos realizar a otimização de execução de tarefas no software como exemplo diminuir a frequência de transmissão de dados quando a carga da bateria atingir $20 \%$.

Com objetivo de utilizar o mínimo de componentes para monitoramento da bateria, foi projetado o circuito utilizando o DS2745, que realiza monitoramento da bateria com um consumo de $70 \mu \mathrm{A}$. Com este circuito integrado são coletados dados de medição de corrente bidirecional com um conversor AD de 16-Bits e mais dois conversores AD de 11-Bits, sendo um para monitoramento da tensão da bateria e outro para temperatura da 
bateria. O processo de monitoramento de corrente é feito por meio da utilização de um resistor de baixo valor, também chamado de resistor shunt.

\subsection{Design dos sensores e transceptores}

A placa MANNA-WUI veem com intuito de ser um nó sensor versátil e algumas aplicações podem usar sensores externos e não ser de interesse os sensores já contidos na placa. Deste modo como visto na Figura 1 o circuito dos sensores e transceptores possuem um transistor NPN que atua como chave, operando na região de saturação ou de corte. A ligação ON/OFF destes sensores e transceptores são realizadas por meios de jumpers contidos na placa como visto na Figura 2.

A utilização desta configuração permite deixar em corte completamente sensores e transceptores que não serão utilizados. Estes componentes podem operar em modo sleep fazendo seu consumo ser baixo, mas este consumo por mínimo que seja será contabilizado na bateria e em aplicações que utilizam apenas um ou dois sensores, este consumo dos componentes hibernados pode ser críticos no desempenho energético.

\section{Custo para montagem do nó sensor}

Os custos para construção e montagem do nó sensor são apresentados na Tabela 2. Os valores são referentes para construção de uma única placa. $O$ custo referente a montagem da placa (soldagem) não são apresentados na Tabela 2, devido o processo ser realizado de forma manual pelo próprio autor.

A categoria suplementos apresentada na Tabela 2 refere-se a um valor global de todos os resistores, capacitores, transistores e conectores utilizados na confecção de uma placa. O custo de fabricação da PCB (Printed Circuit Board) foi de $\$ 47,6$ para 15 placas. $\mathrm{O}$ valor apresentado na Tabela 2 é referente a uma placa. Todos os valores estão expressos em moeda americana (dólar). O custo total para montagem de um dispositivo foi de $\$$ 49,2. Esse valor pode ser reduzido em uma produção em larga escala.

Tabela 2. Valores para construção de uma placa.

\begin{tabular}{|l|c|c|}
\hline \multicolumn{1}{|c|}{ Componentes } & Quantidade & Valor (US \$) \\
\hline STM32F407VGT6 & 1 & 11,72 \\
\hline SX1276 & 1 & 8,00 \\
\hline ESP32-DOWDQ6 & 1 & 3,80 \\
\hline BME280 & 1 & 6,00 \\
\hline MPU9250 & 1 & 2,30 \\
\hline APDS9960 & 1 & 2,50 \\
\hline DS4520 & 1 & 2,70 \\
\hline FT232R & 1 & 4,00 \\
\hline PCB & - & 3,18 \\
\hline Suplementos & - & 5,00 \\
\hline Total & & 49,2 \\
\hline
\end{tabular}

\subsection{Implementação do algoritmo}

O software que realiza o controle do nó sensor foi desenvolvido utilizando a linguagem de programação C. A placa do dispositivo desenvolvido conta com um cristal de 32,768 $\mathrm{kHz}$ exclusivo para funcionamento do RTC (Real Time Clock). No momento em que o nó sensor se conecta a rede, o gateway envia uma resposta e sincroniza o relógio do 
dispositivo. Assim, o novo integrante da rede recebe também as configurações de funcionamento, informando o tempo de cada transmissão. Os jumpers de ON/OFF dos sensores e transmissores, foram conectados cada um em um pino do MCU permitindo realizar o processo de liga e desliga por meio do software.

Não utilizou-se o protocolo LoRaWAN, mas foi elaborado um protocolo baseado em LoRa-MAC para realização dos testes, que contam com 29-bytes, compostos pelo endereço do nó sensor de destino (4-bytes), seguido pelo endereço de origem (4-bytes), finalizando com informações dos sensores e bateria (21-bytes).

A Figura 3 apresenta o fluxograma de funcionamento do sensor após o mesmo já estar conectado a rede. O dispositivo permanece no modo sleep até o momento de realizar uma transmissão. O sistema de RTC gera um alarme de wake up (acordar) a cada minuto. Ao entrar no estado ativo, o sistema liga os sensores e o transceptor e em seguida inicia a aquisição de dados dos sensores. Neste momento todos os sensores são lidos e as informações necessárias são processadas para, em seguida, serem transmitidas. Após sua transmissão, os sensores e transceptores são desativados e MCU retornam para o estado sleep até iniciar um novo ciclo de medição.

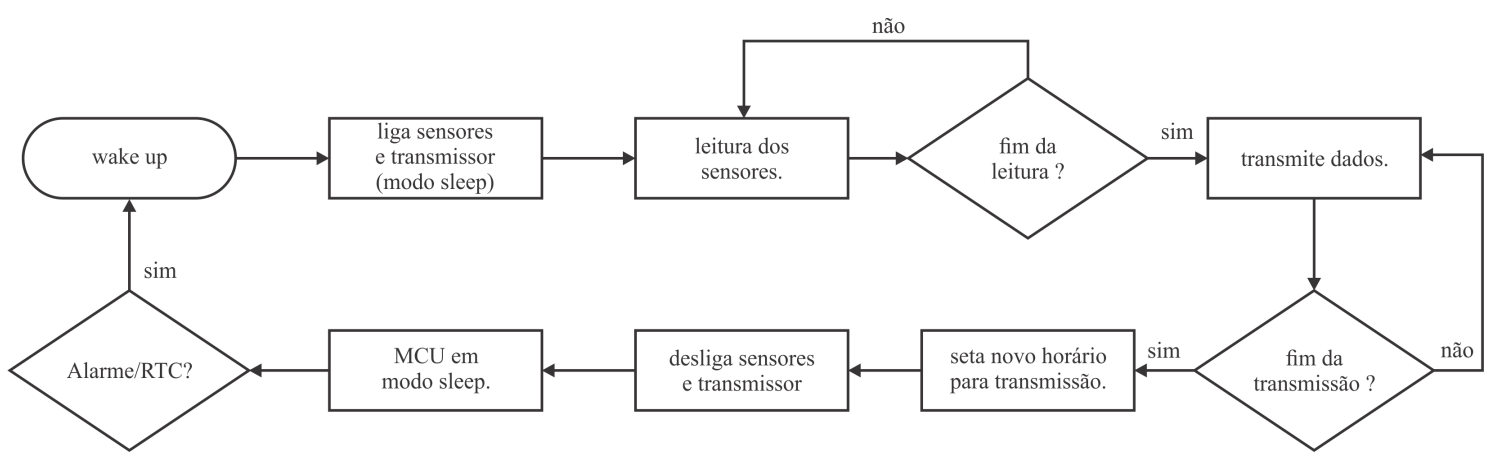

Figura 3. Fluxograma do funcionamento do nó sensor.

\section{Avaliação da performance do MANNA-WUI}

De modo a avaliar a performance do MANNA-WUI, foram conduzidos experimentos em ambientes reais, urbanos e não-urbanos. Os testes realizados focam o desempenho durante a transferência de dados em ambiente urbanos e não urbanos, avaliando os indicadores RSSI (Received Signal Strength Indication), SNR (Signal-to-Noise Ratio) e eficiência energética. As seções a seguir apresentam a configuração estabelecida para os experimentos e os resultados obtidos.

\subsection{Configuração do Experimento em Cenário Urbano}

Foi utilizado a topologia da rede tipo estrela e os testes foram realizados com 3 nós sensores, sendo que um atua como gateway. O transceptor LoRa foi configurado para operar na faixa de $915 \mathrm{MHz}$, BW (Bandwidth) de $125 \mathrm{kHz}$, SF (Spreading Factor) de 8, EC (Error Correction Code) de 4/6 e potência de saída da antena de $20 \mathrm{dBm}$.

O gateway foi instalado no quarto andar de um edifício ficando a uma altura referente ao solo de 16 metros. Ao seu redor haviam prédios com maior números de andares e diversas árvores. A comunicação era estabelecida a cada 1 minuto. O dispositivo transmitia um pacote de 29-Bytes, com informações dos sensores e status da bateria. Os relógios 
dos dispositivos eram sincronizados no momento em que o nó se conecta pela primeira vez com o gateway.

Os nós sensores foram instalados a uma altura de 1 metro referente ao solo. A Figura 4 (a) apresenta a configuração dos nós sensores. O sensor (Nó B) mais distante estava a 728,66 metros e o mais próximo (Nó A) a 606,71 metros. As distâncias apresentadas foram estimadas utilizando o Google Maps.

\subsection{Configuração do Experimento em Cenário Não-Urbano}

No teste realizado no cenário não-urbano, o gateway se encontrava a uma altitude de 12,5 m no topo de um prédio de 2 andares. As configurações BW, SF, EC e potência de saída do transceptor LoRa não foram alteradas, sendo as mesmas para o cenário urbano (5.1). O processo de comunicação era estabelecido a cada 1 minuto. Neste, o nó também transmitia um pacote com 29-Bytes.

A Figura 4 (b) apresenta a configuração da disposição dos nós sensores. O Nó A é o que se encontra mais distante do gateway, cerca de $18,5 \mathrm{Km}$. O Nó B se encontra a uma distância de $16 \mathrm{Km}$. Inicialmente, os nós A e B foram instalados a uma altitude de 1,7m. O nó A realizou troca de dados com gateway. Devido a grande quantidade de vegetação ao redor do local em que o nó B estava instalado, foi necessário aumentar a altitude do nó sensor B em 2,5m, chegando a 4,2m de altitude em relação ao solo, para se comunicar com o gateway.

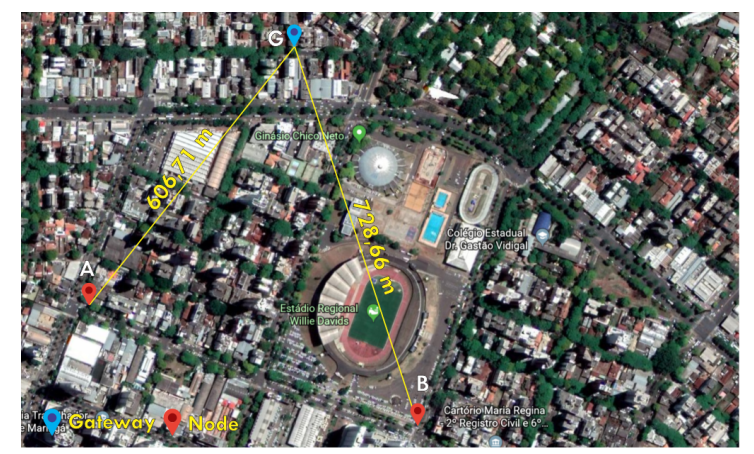

a

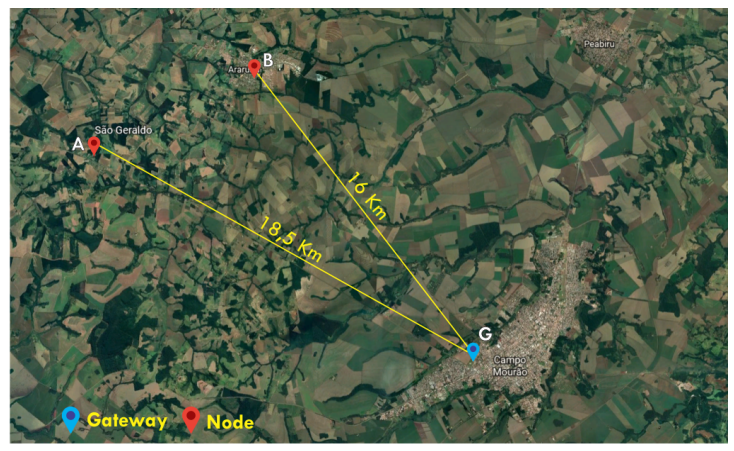

b

Figura 4. Mapa referente do local onde se encontram instalados os nós em ambiente urbano (a) e não urbano (b).

\subsection{Resultados Obtidos}

O comportamento do RSSI e SNR dos nós A e B para a configuração apresentada na Subseção 5.1 são apresentados na Figura 5 em forma de gráfico, no decorrer de 25 transmissões. Analisando a intensidade do sinal recebido (RSSI) o Nó A apresenta intensidade mais fraca em relação ao Nó B que se encontrava a uma maior distância, ficando com um RSSI médio de -117 dBm e SNR de $-12 \mathrm{~dB}$.

O Nó B apresenta um RSSI médio de -106 dBm e SNR de -10 dB. Observamos que essa diferença do RSSI foi gerada devido a área entre o gateway até o Nó B possuir menor vegetação e edifícios como mostra a Figura 4 (a). No decorrer das 25 transmissões, todos os pacotes foram recebidos com sucesso. 
O teste realizado em cenário não-urbano para configurações apresentadas na Subseção 5.2 a análise do RSSI e SNR foram para uma sequência de 50 transmissões devido a distâncias em que se encontravam os nós e possíveis perdas de pacotes no decorrer das transmissões. A Figura 6 apresenta o gráfico resultante das medições. No decorrer das 50 transmissões não houve perda de pacotes, mas dois pacotes foram recebidos corrompidos. Um logo no início sendo na sexta transmissão com RSSI de -132 dBm e SNR $-13 \mathrm{~dB}$. E novamente na trigésima sexta transmissão com RSSI de $-133 \mathrm{dBm}$ e SNR -13 $\mathrm{dB}$. Ao conferir o pacote enviado com o recebido, observamos que nos dois casos os bytes de 0 a 12 foram recebidos com sucesso e 12 a 20 foram corrompidos.

Sabendo que o Nó A está mais distante, em uma altitude referente ao nível do mar mais elevada e no decorrer das 50 transmissões todas foram bem-sucedidas. Constatou-se que o principal empecilho que agrava as transmissões são os obstáculos naturais como a vegetação em geral e nível do solo, ajudam a obstruir o sinal diminuindo sua intensidade como mostrado no Gráfico RSSI/SNR Nó B apresentado na Figura 6 (b) a intensidade chega a $-133 \mathrm{dBm}$.

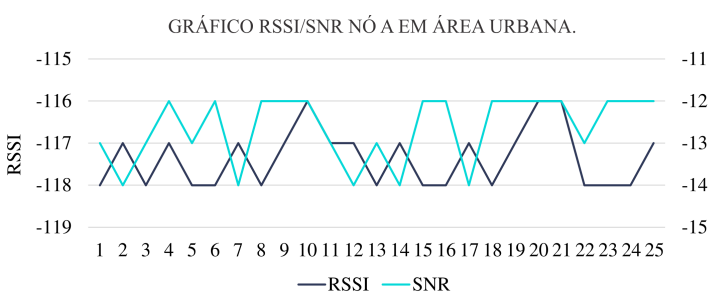

a

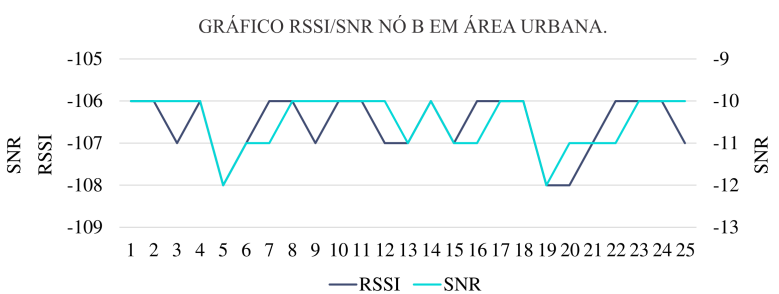

b

Figura 5. Comportamento do RSSI e SNR em ambiente urbano no decorrer de 25 transmissões.

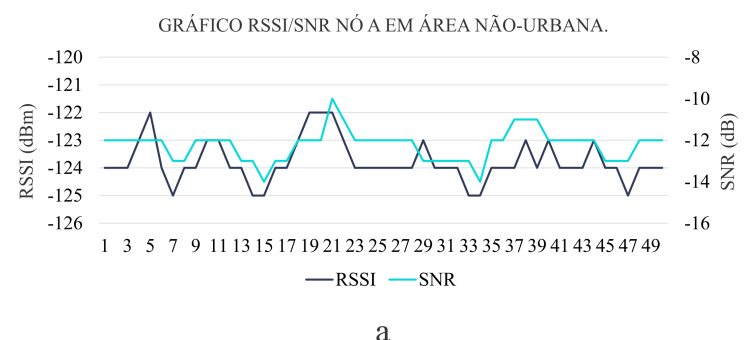

a

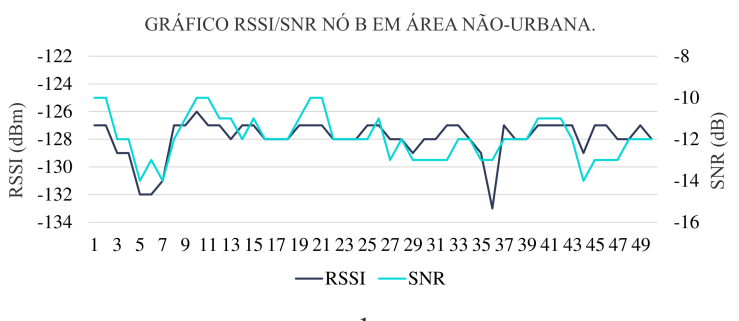

$\mathrm{b}$

Figura 6. Comportamento do RSSI e SNR em ambiente não urbano no decorrer de 50 transmissões.

A potência de consumo do nó sensor durante um clico de operação é mostrado na Figura 7. As medições foram realizadas através do sistema de monitoramento da bateria disponível no próprio dispositivo mencionado na Seção 3.1. Uma bateria de $3400 \mathrm{mAh}$ e tensão 3,7V fornecia energia ao sistema.

Como esperado o modo de maior consumo de corrente é no momento de transmissão de dados chegando a ter um consumo de $422 \mathrm{~mW}$. No momento em que o microcontrolador está ativo seu consumo é de $9,9 \mathrm{~mW}$ e na aquisição de dados dos sensores a corrente do sistema é de $21 \mathrm{~mW}$. No último modo todos os sensores e transmissores estão desligados por meio chave ON/OFF e o microcontrolador entra no modo sleep e a 
corrente drenada da bateria é de $13,2 \mu \mathrm{W}$. A chave ON/OFF permite ligar e desligar os sensores e transceptores fazendo com que não tenha o consumo do modo sleep, somente do MCU.

Gráfico de consumo para execução de 1 ciclo.

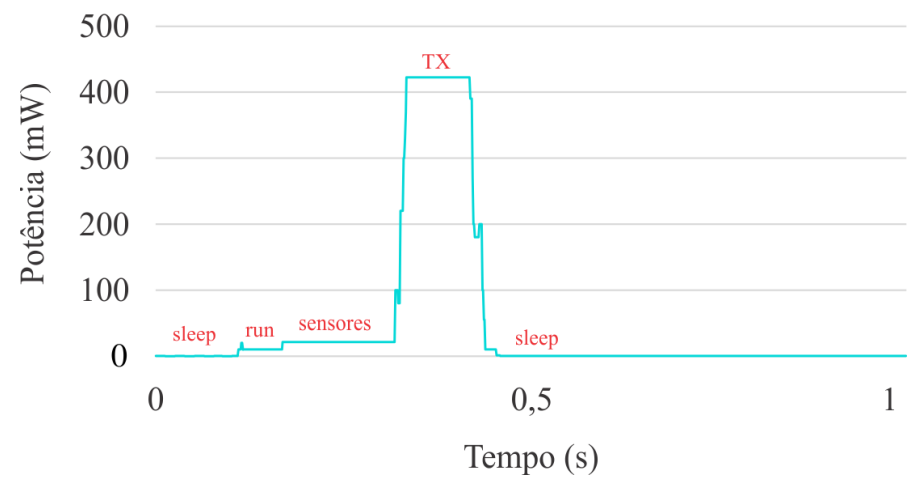

Figura 7. Consumo do nó sensor durante a execução de 1 ciclo.

A Tabela 3 apresenta o consumo de cada subsistemas do nó sensor. Para o gráfico da Figura 7 o sistema Wi-Fi estava desligado completamente através da chaves ON/OFF mencionados na Seção 3.2 e por este motivo não existe consumo. Observamos na Tabela abaixo que os sistemas que são utilizados para realização de transmissão de dados são justamente os que causam mais impacto na vida útil da bateria devido a potência dos mesmos.

Tabela 3. Potências do subsistemas do nó sensor.

\begin{tabular}{|l|l|c|}
\hline \multicolumn{1}{|c|}{ Sistemas } & \multicolumn{1}{c|}{ Modo } & Potência \\
\hline MCU & RUN & $9,9 \mathrm{~mW}$ \\
\hline MCU & SLEEP & $13,2 \mu \mathrm{W}$ \\
\hline SENSORES & ON & $10,5 \mathrm{~mW}$ \\
\hline SENSORES & OFF & $26,4 \mu \mathrm{W}$ \\
\hline LoRa & TX & $396 \mathrm{~mW}$ \\
\hline LoRa & RX & $46,2 \mathrm{~mW}$ \\
\hline LoRa & SLEEP & $3,3 \mu \mathrm{W}$ \\
\hline Wi-Fi & ON & $650 \mathrm{~mW}$ \\
\hline Wi-Fi & SLEEP & $330 \mu \mathrm{W}$ \\
\hline
\end{tabular}

\section{Conclusão}

Este trabalho introduziu e avaliou a performance de um dispositivo para o paradigma da Internet das Coisas denominado MANNA-WUI. O nó sensor foi desenvolvido considerando as variadas possibilidades de aplicações para IoT, propondo a utilização de um microcontrolador mais robusto do que os que são utilizados em aplicações do gênero, mas mantendo o foco no baixo consumo e transmissão a longa distâncias.

O nó sensor projetado apresentou um bom desempenho para transmissões a longas distâncias em ambientes não urbanos conseguindo realizar troca de dados a 18,5km. O consumo energético do dispositivo foi aceitável, mas quando falamos de nó sensores que 
necessitam ter uma durabilidade da bateria de meses ou anos, o fator que mais afeta o consumo energético do dispositivo é o meio de transmissão de dados.

Em trabalhos futuros pretende-se conectar uma câmera de baixo custo no nó sensor. Devido a escolha de um microcontrolador mais robusto, o mesmo fornece uma interface DCMI (Digital Camera Interface) possibilitando realizar o pré processamento e transmitindo apenas informações desejadas de ambientes e processos que necessitam de monitoramento, realizar comparações de desempenho do dispositivo MANAA-WUI com nó sensores disponíveis no mercado e assim avaliar seu desempenho.

\section{Agradecimentos}

O presente trabalho foi realizado com apoio recebido pelo grupo de pesquisa MANNA Team, Conselho Nacional de Desenvolvimento Científico e tecnológico - CNPq - Brasil e Coordenação de Aperfeiçoamento de Pessoal de Nível Superior - Brasil (CAPES) Código de Financiamento 001.

\section{Referências}

Adam, A. H., Tamilkodi, R., and Madhavi, K. V. (2019). Low-cost green power predictive farming using iot and cloud computing. In 2019 International Conference on Vision Towards Emerging Trends in Communication and Networking (ViTECoN), pages 1-5.

Addabbo, T., Fort, A., Mecocci, A., Mugnaini, M., Parrino, S., Pozzebon, A., and Vignoli, V. (2019). A lora-based iot sensor node for waste management based on a customized ultrasonic transceiver. In 2019 IEEE Sensors Applications Symposium (SAS), pages $1-6$.

Baldovino, R. G., Valenzuela, I. C., and Dadios, E. P. (2018). Implementation of a low-power wireless sensor network for smart farm applications. In 2018 IEEE 10th International Conference on Humanoid, Nanotechnology, Information Technology,Communication and Control, Environment and Management (HNICEM), pages $1-5$.

BOSCH (2018). BME280 - Combined Humidity and Pressure Sensor. [Online; accessed 15-August-2019].

Bouguera, T., Diouris, J., Chaillout, J., and Andrieux, G. (2018). Energy consumption modeling for communicating sensors using lora technology. In 2018 IEEE Conference on Antenna Measurements Applications (CAMA), pages 1-4.

Dallas Semiconductor (2009). Battery Monitor. [Online; accessed 15-August-2019].

Derhamy, H., Eliasson, J., Delsing, J., and Priller, P. (2015). A survey of commercial frameworks for the internet of things. In 2015 IEEE 20th Conference on Emerging Technologies Factory Automation (ETFA), pages 1-8.

Duangsuwan, S., Takarn, A., Nujankaew, R., and Jamjareegulgarn, P. (2018). A study of air pollution smart sensors lpwan via nb-iot for thailand smart cities 4.0. In 2018 10th International Conference on Knowledge and Smart Technology (KST), pages 206-209.

Edward Jero, S. and Balaji Ganesh, A. (2011). Pic18lf4620 based customizable wireless sensor node to detect hazardous gas pipeline leakage. In 2011 International Conference on Emerging Trends in Electrical and Computer Technology, pages 563-566. 
ESPRESSIF (2019). ESP32 Series. [Online; accessed 08-September-2019].

Fitriawan, H., Susanto, M., Arifin, A. S., Mausa, D., and Trisanto, A. (2017). Zigbee based wireless sensor networks and performance analysis in various environments. In 2017 15th International Conference on Quality in Research (QiR) : International Symposium on Electrical and Computer Engineering, pages 272-275.

Gartner (2018). Gartner identifies top 10 strategic iot technologies and trends. [Online; accessed 20-March-2020].

InvenSense (2016). MPU-9250 - Product Specification. [Online; accessed 15-August2019].

Jörke, P., Falkenberg, R., and Wietfeld, C. (2018). Power consumption analysis of nb-iot and emtc in challenging smart city environments. In 2018 IEEE Globecom Workshops (GC Wkshps), pages 1-6.

Kjellby, R., Cenkeramaddi, L. R., Johnsrud, T. E., Jevne, G., Løtveit, S. E., Beferull Lozano, B., and Joshi, S. (2018). Design and prototype implementation of long-range self-powered wireless iot devices. In 2018 IEEE International Symposium on Smart Electronic Systems (iSES) (Formerly iNiS), pages 215-218.

Lee, I. and Lee, K. (2015). The internet of things (iot): Applications, investments, and challenges for enterprises. Business Horizons, 58(4):431 - 440.

Lin, P. and Wang, K. (2017). A smart sensor node design for efficient communication in zigbee wireless sensor networks. In 2017 International Conference on Information, Communication and Engineering (ICICE), pages 366-369.

Magno, M., Aoudia, F. A., Gautier, M., Berder, O., and Benini, L. (2017). Wulora: An energy efficient iot end-node for energy harvesting and heterogeneous communication. In Design, Automation Test in Europe Conference Exhibition (DATE), 2017, pages $1528-1533$.

Mayer, P., Magno, M., Brunner, T., and Benini, L. (2019). Lora vs. lora: In-field evaluation and comparison for long-lifetime sensor nodes. In 2019 IEEE 8th International Workshop on Advances in Sensors and Interfaces (IWASI), pages 307-311.

SEMTECH (2019). Datasheet - SX1276/77/78/79 - $137 \mathrm{MHz}$ to $1020 \mathrm{MHz}$ Low Power Long Range Transceiver. [Online; accessed 08-September-2019].

Serikul, P., Nakpong, N., and Nakjuatong, N. (2018). Smart farm monitoring via the blynk iot platform : Case study: Humidity monitoring and data recording. In 2018 16th International Conference on ICT and Knowledge Engineering (ICT KE), pages $1-6$.

Tokmakov, D., Asenov, S., and Dimitrov, S. (2019). Research and development of ultralow power lorawan sensor node. In 2019 IEEE XXVIII International Scientific Conference Electronics (ET), pages $1-4$.

Wang, J., Su, J., and Hua, R. (2019). Design of a smart independent smoke sense system based on nb-iot technology. In 2019 International Conference on Intelligent Transportation, Big Data Smart City (ICITBS), pages 397-400. 